\title{
Antiangiogenic effects of recombinant human endostatin in lung cancers
}

\author{
LANG HE $^{1-3^{*}}, \mathrm{CHAOFEN} \mathrm{ZHAO}^{4 *}$, YUNXIANG LI ${ }^{* *}$, GUOCHENG DU $^{6 *}$, KANG LIU $^{7}$, \\ DANDAN CUI ${ }^{1 *}$, LINA TANG $^{8}, \mathrm{XUN}^{2} \mathrm{WU}^{8}, \mathrm{SHIMIN} \mathrm{WEN}^{2}$ and $\mathrm{HONG} \mathrm{CHEN}^{9}$
}

\begin{abstract}
${ }^{1}$ Department of Oncology, The Fifth People's Hospital of Chengdu, North Sichuan Medical College, Chengdu, Sichuan 611130; ${ }^{2}$ Cancer Center, The Second Clinical Medical College of North Sichuan Medical College, Nanchong, Sichuan 637000; ${ }^{3}$ Cancer Center, Suining Central Hospital, Suining, Sichuan 629000; ${ }^{4}$ Department of Medical Oncology, Affiliated Hospital of Guizhou Medical University, Guizhou Cancer Hospital, Guiyang, Guizhou 550004; Departments of ${ }^{5}$ Urology and ${ }^{6}$ Galactophore, The Second Clinical Medical College of North Sichuan Medical College; ${ }^{7}$ Institute of Tissue Engineering and Stem Cells; ${ }^{8}$ Department of Clinical Medicine, North Sichuan Medical College, Nanchong, Sichuan 637000;

${ }^{9}$ Tumor Department of TCM, Sichuan Cancer Hospital and Institute, Chengdu, Sichuan 610000, P.R. China
\end{abstract}

Received June 15, 2016; Accepted August 22, 2017

DOI: $10.3892 / \mathrm{mmr} .2017 .7859$

\begin{abstract}
Antiangiogenic therapy, as a new anticancer method, can improve the anticancer effect of traditional therapies. Different antiangiogenic drugs may have different vascular normalization time windows. Whether the antiangiogenic treatment is within the vascular normalization time window is very important in the treatment of cancers. Previous studies have indicated that recombinant human endostatin (rh-ES) can transiently normalize tumor microvessels. Yet the molecular mechanism and the time window of rh-ES remains unclear. The aim of the present study was to explore the optimal time window and molecular mechanism of rh-ES in inhibiting Lewis lung cancer (LLC). By comparatively accessing the changes in microvascular and hypoxic conditions of tumors in host mice treated with rh-ES or saline for different days, the authors aimed to investigate the best administration time of rh-ES treatment on human lung cancers and obtain a better understanding concerning the involved molecular mechanism. A total of 40 C57/BL6 mice with LLC xenografts were randomly divided into normal saline (NS) and rh-ES groups
\end{abstract}

Correspondence to: Dr Shimin Wen, Cancer Center, The Second Clinical Medical College of North Sichuan Medical College, 97 South Renmin Road, Nanchong, Sichuan 637000, P.R. China E-mail: wenshimin_sc@sina.com

Dr Hong Chen, Tumor Department of TCM, Sichuan Cancer Hospital and Institute, Section 4, 55 South Renmin Road, Chengdu, Sichuan 610000, P.R. China

E-mail: chenhongdr@163.com

*Contributed equally

Key words: recombinant human endostatin, Lewis lung cancer, vascular normalization, regulator of G-protein signaling 5, carbonic anhydrase 9
(20 mice/group). $0.2 \mathrm{ml} \mathrm{NS}$ or $5 \mathrm{mg} / \mathrm{kg}$ rh-ES were administrated via intraperitoneal injection (i.p.) into each mouse each day during the 9-day experiment. A total of 5 mice from each group were sacrificed at day 2, 4, 6 or 9. CA9 and RGS5 expression levels of both groups were compared using immunohistochemistry, reverse transcription-quantitative polymerase chain reaction and ELISA. Rh-ES caused vascular normalization and improved hypoxia at days 4 and 6. Compared with the control (NS) group, both CA9 and RGS5 expression in rh-ES group were significantly decreased at days 4 and $6(\mathrm{P}<0.05)$, while no significant change between two groups was observed at days 2 and 9. Rh-ES can induce transient tumor vascular normalization and improves tissue hypoxia in LLC tumors. The vascular normalization window is accompanied by the reduction in RGS5 and CA expression.

\section{Introduction}

Previously, it was believed that antiangiogenic drugs inhibit tumors by promoting degradation of tumor blood vessels, causing ischemia and hypoxia in the tumor, leading to tumor cell death (1). According to this theory, anti-angiogenic drugs would antagonize the effect of other cancer treatments, such as chemotherapy, because the lack of oxygen and blood supply impedes the delivery of other drugs. However, the truth is that the antiangiogenic drugs actually enhance the efficacy of radiotherapy and chemotherapy. Therefore, antiangiogenic drugs may inhibit tumors by some other mechanisms. In 2004, Lin and Sessa (2) first proposed the 'time window' concept for tumor angiogenesis. They believed that there is a certain period, during which antiangiogenic drugs have the greatest anti-tumor effect. In this time window, the newly formed tumor vessels tended to be normal in vascular endothelial maturation, morphological regulation and tight junctions, and thus, anticancer drugs can easily reach the tumor cells, directly damaging tumor cell DNA and inhibiting vascular endothelial cell proliferation. 
The time window of tumor vessel normalization is specifically marked by improved tumor hypoxic status and change in pericyte coverage $(1,3)$. Generally, pericytes in the tumor vascular bed display obviously abnormal structure and the pericyte coverage is low (4). Pericyte maturation and blood vessel normalization take place following the depletion of regulator of G-protein signaling 5 (RGS5), one of the proteins that reflects pericyte coverage $(5,6)$. Hypoxia, a common phenomenon in human tumors (7), can cause tumors to become resistant to therapy and lead to tumor progression (8). Carbonic anhydrase 9 (CA9), a transmembrane zinc metalloenzyme, can be induced by hypoxia and promoting aggressive behaviors of tumors by helping maintain normal intracellular $\mathrm{pH}$ and preventing apoptosis in a hypoxic environment (9).

Endostatin is the $20 \mathrm{kDa}$-terminal fragment of collagen XVIII that is primarily located in the basement membrane of blood vessels. It was first described by O'Reilly et al (10) in 1997 and was licensed by EntreMed. In spite of the fact that preclinical and clinical studies on tumor suppression were very positive, EntreMed abandoned phase III clinical trials due to an insufficient supply of endostatin. Nevertheless, endostatin development was continued by scientists in China and recombinant human endostatin (rh-ES, with the trade name Endostar) has been approved by the China Food and Drug Administration. The use of it in combination with chemotherapy has been suggested by the National Comprehensive Cancer Network (NCCN) Guidelines to treat non-small cell lung cancer (NSCLC). However, the optimal administration time of rh-ES and its molecular mechanism still remain unclear. In the current study, by using a Lewis lung cancer (LLC) tumor model, the authors aimed to identify the optimal administration time of rh-ES in cancer treatment and its possible molecular mechanism.

\section{Materials and methods}

Ethical statement. The present study was approved by the Ethics Committee of North Sichuan Medical College (Nanchang, China).

Cell culture and animal model. Mouse LLC tumor cells were purchased from the State Key Laboratory of Cancer Biotherapy at Sichuan University (Chengdu, China). The 6-8 week-old female mice with a body weight of 15-20 g were purchased from the Laboratory Animal Center of North Sichuan Medical College (Nanchong, China). LLC cells at logarithmic growth phase were collected as a $1 \times 10^{6}$ cells $/ \mathrm{ml}$ single cell suspension. Cells $(0.2 \mathrm{ml})$ were injected subcutaneously into the left armpit of each C57/BL6 mouse. The growth and tumor formation in the mice was observed and recorded.

Animal treatment and tumor sample collection. Treatment started when the LLC tumors reached a diameter of $6 \mathrm{~mm}$. A total of 40 mice were randomly split into two equal groups: NS and rh-ES. Mice in the NS group were intraperitoneally injected with $0.2 \mathrm{ml} 0.9 \%$ normal saline daily for up to 9 days, while mice in rh-ES group were intraperitoneally injected with $5 \mathrm{mg} / \mathrm{kg}$ rh-ES (Shandong Simcere-Medgenn
Bio-Pharmaceutical Co., Ltd., Yantai, China) daily for up to 9 days. A total of 5 mice from each group were sacrificed at days 2, 4, 6 and 9 following treatment. Each tumor sample was split into two parts. One part was snap-frozen within $30 \mathrm{~min}$ following animal sacrifice and stored at $-80^{\circ} \mathrm{C}$ for further RNA and protein analyses. The other part was fixed with $4 \%$ paraformaldehyde and dehydrated, and embedded in paraffin for immunohistochemical analysis.

Immunohistochemistry (IHC). Sections $(5 \mu \mathrm{m})$ were prepared from paraformaldehyde-fixed, paraffin-embedded tissues and were used for immunocytochemistry. The CD31 antibody (cat no. ab28364; dilution, 1:500) was obtained from Abcam (Cambridge, MA, USA) and incubated at $37^{\circ} \mathrm{C}$ for $2 \mathrm{~h}$. RGS5 (cat no. ab138019; Abcam, Shanghai, China) and CA9 (cat no. NB100-417; Novus Biologicals, LLC, Littleton, CO, USA) antibodies were used at a $1: 100$ dilution at $37^{\circ} \mathrm{C}$ for $2 \mathrm{~h}$. The bound antibody was visualized with SABC IHC kit (Santa Cruz Biotechnology, Inc., Dallas, TX, USA). The positive RGS5 signal was located in the cytoplasm and had a color of light yellow to brown, while the CA9 signal was located on the cell membrane and had a color of brown. The authors followed the criteria used by Rahman et al (11) and used the gray color intensity of positive cells as the indicator of signal strength: 0 , negative; 1 , weak positive; 2 , positive; 3 , strongly positive. The staining range ( $\%$ of positive cells) was scored from $0-4$ : 0 , negative; $1,1-25 \%$ positive cells; $2,26-50 \% ; 3,51-75 \% ; 4$, $76-100 \%$. Five non-overlapping fields (magnification, $x 400$ ) were randomly selected and calculated the percentage of positive cells in 1,000 tumor cells.

Reverse transcription-quantitative polymerase chain reaction $(R T-q P C R)$. Total RNA samples were extracted from tissues using Total RNA extraction kit (Omega Bio-Tek, Inc., Norcross, GA, USA). cDNA samples were synthesized using a cDNA synthesis kit (Thermo Fisher Scientific, Inc., Waltham, MA, USA) $\left(42^{\circ} \mathrm{C}, 60 \mathrm{~min} ; 70^{\circ} \mathrm{C}, 5 \mathrm{~min}\right)$ and the cDNA used for RT-qPCR (Stage 1: $95^{\circ} \mathrm{C}, 30 \mathrm{sec}$; Stage 2: $95^{\circ} \mathrm{C}, 5 \mathrm{sec} ; 60^{\circ} \mathrm{C}, 34 \mathrm{sec}$; repeated 40 times. Stage $3: 95^{\circ} \mathrm{C}$, $15 \mathrm{sec} ; 60^{\circ} \mathrm{C}, 60 \mathrm{sec}$ and $95^{\circ} \mathrm{C}, 15 \mathrm{sec}$ ). Primers were purchased from Shanghai Generay Biotech Co., Ltd. (Shanghai, China). Sequences are as follows: GAPDH forward, 5'-AGAAGGTGG TGAAGCAGGCATC-3' and reverse, 5'-CGAAGGTGGAAG AGTGCGAGTTG-3'; RGS5 forward, 5'-ATCAAAATGGCG GAGAAGGCAAA-3' and reverse, 5'-CACAAAGCGGGG CAGAGAATC-3'; CA9 forward, 5'-TGTGGGGACCTCGTG ATTCTCG-3' and reverse, 5'-TGGACTGGCTCAGGGCTG CTAT-3'. The relative levels of gene expression were quantified by using the comparative $\mathrm{Cq}$ method (12).

ELISA. Tumor tissues were homogenized using $1 \mathrm{X}$ cell lysis buffer (cat no. 9803; Cell Signaling Technology, Inc., Danvers, MA, USA) and centrifuged at $-4^{\circ} \mathrm{C}$ and $12,000 \mathrm{x}$ g for $15 \mathrm{~min}$. The supernatant was harvested and tested using ELISA kits (cat nos. MBS2604739 and MBS939749; MyBiosource, Inc., San Diego, CA, USA) for RGS5 and CA9 following manufacturer's protocols. A total volume of $100 \mu \mathrm{l}$ blank, standards, and tumor samples were added into wells. Optical density was measured at $450 \mathrm{~nm}$. Results are expressed as $\mathrm{ng} / \mathrm{ml}$ of homogenizing buffer. 


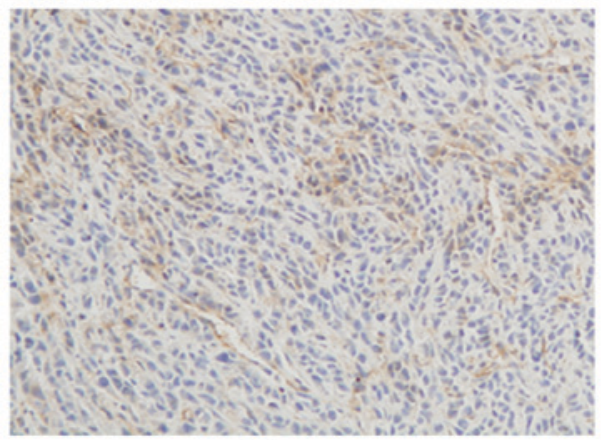

2 days

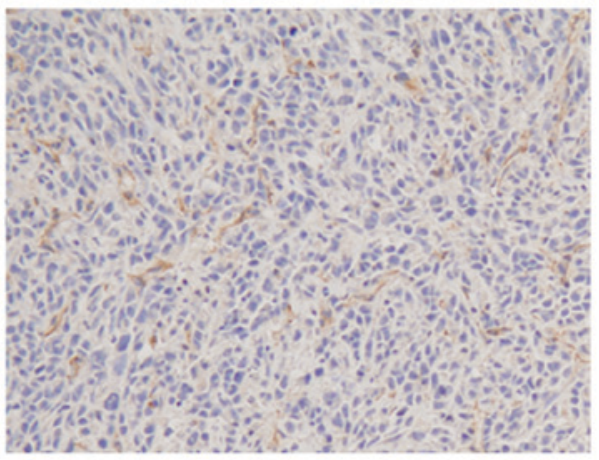

6 days

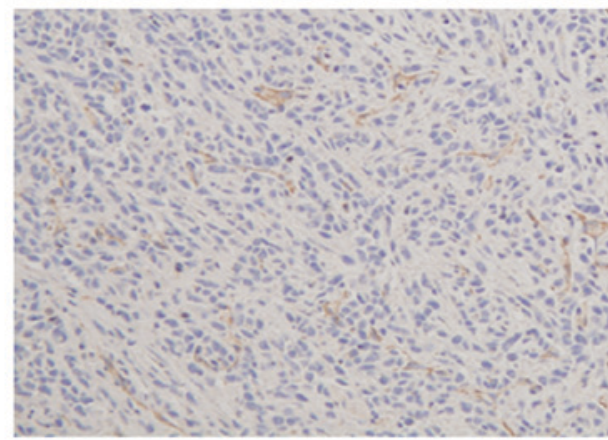

4 days

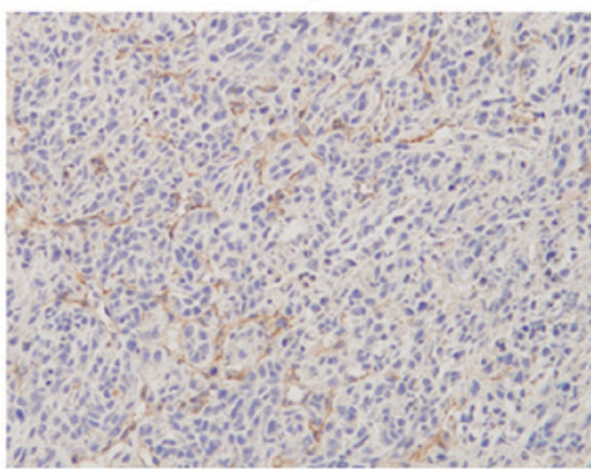

9 days

Figure 1. Representative images presenting CD31 (a marker for microvessels) immunohistochemistry staining in Lewis lung cancer tumors following treatment with recombinant human endostatin for days 2, 4, 6 and 9. Magnification x400.

Table I. Microvessel density value in tumor tissues.

\begin{tabular}{lcc}
\hline $\begin{array}{l}\text { Days following } \\
\text { injection }(\mathrm{n})\end{array}$ & $\mathrm{n}$ & $\begin{array}{c}\text { Microvessel density } \\
\left(\text { cells } / \mathrm{mm}^{2}\right)\end{array}$ \\
\hline 2 & 5 & $23.10 \pm 3.26$ \\
$4^{\mathrm{a}}$ & 5 & $10.51 \pm 3.12$ \\
$6^{\mathrm{a}}$ & 5 & $9.39 \pm 1.94$ \\
9 & 5 & $21.86 \pm 3.25$
\end{tabular}

${ }^{\text {ap }}<0.05$ (Rh-ES vs. NS). Values are presented as mean \pm standard deviation.

Statistics. Data are presented as the mean \pm standard deviation. The significance between two groups was tested using Student's t-test (two tailed) and $\mathrm{P}<0.05$ was considered to indicate a statistically significant difference. SPSS software (version, 13.0; SPSS, Inc., Chicago, IL, USA) was used for statistical analysis.

\section{Results}

LLC tumor formation. The tumor formation rate in C57/BL6 female mice was $100 \%$. On day 10 , following subcutaneous injection of LLC cells, tumor xenografts grew to a size of $\sim 6 \mathrm{~mm}$ in diameter. The tumors were hard and spherical, and displayed poor activity and expansive growth.

Rh-ES causes transient LLC tumor vascular normalization and improves tumor tissue hypoxia. To examine the changes in tumor vascularization, the authors performed immunohistochemical staining for CD31, a microvessel marker, on LLC tumors treated with rh-ES for 2, 4, 6 and 9 days. It was reported that LLC tumors treated with rh-ES for 4 or 6 days presented lower CD31 expression than those treated for 2 or 9 days. The authors calculated microvessel density as a ratio of microvessel number to the area of the image to measure CD31 immunoreactivity. The data indicated a significant reduction in the vascularity of the LLC tumor xenografts following treatment with rh-ES for 4 or 6 days. In contrast, no significant change was observed for LLC tumors treated with rh-ES for 2 days or 9 days (Fig. 1 and Table I).

Immunohistochemical analysis demonstrates the reduction of RGS5 and CA9 protein expression in tumor cells at $d 4$ and d6 of treatment, but not at $d 2$ and $d 9$. RGS5 is a master regulator for tumor vascular normalization. CA9 is a biomarker of tumor hypoxia. To test whether rh-ES can cause a change in the expression of these two proteins, using immunohistochemical analysis, the authors examined the RGS5 and CA9 protein expression levels in LLC tumors treated with rh-ES or saline for 2, 4, 6 and 9 days. It was determined that, compared with the NS group, both RGS5 and CA9 levels in rh-ES group were significantly lower at days 4 and $6(\mathrm{P}<0.05)$, while there was no significant change between two groups at days 2 and 9 (Figs. 2-5 and Tables II and III).

$R T$-qPCR analysis demonstrates a reduction of RGS5 and CA9 mRNA levels in tumor cells at days 4 and 6 of treatment, but not at days 2 and 9. To confirm that rh-ES can cause a reduction in the expression of RGS5 and CA9, the authors 


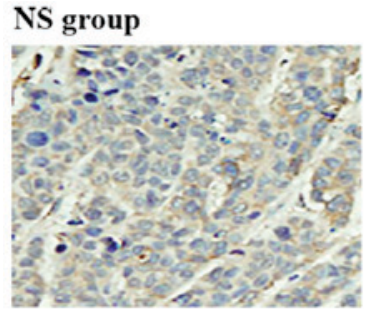

2 days

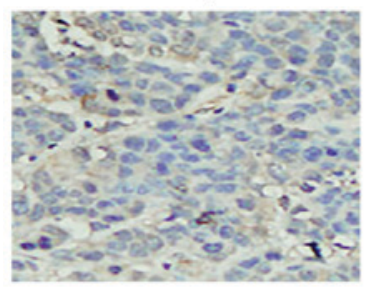

6 days

\section{rh-ES group}

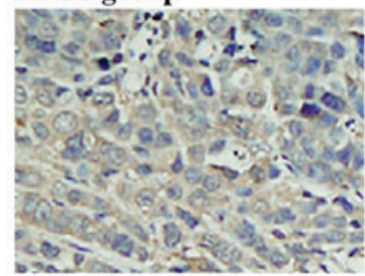

2 days

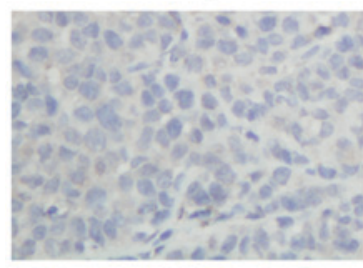

6 days

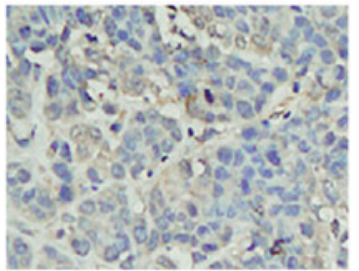

4 days

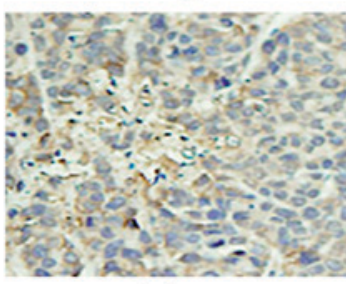

9 days

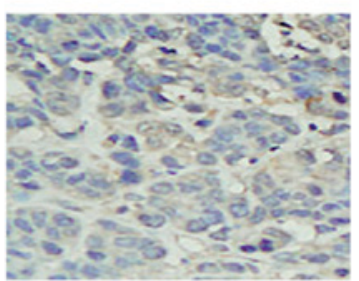

4 days

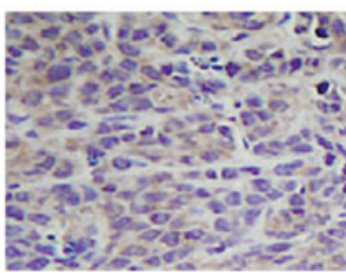

9 days
Figure 2. Representative images showing RGS5 (a marker for tumor vascular normalization) immunohistochemistry staining in Lewis lung cancer tumors after treatment with rh-ES or NS for days 2, 4,6 and 9. The positive RGS5 signals were located in the cytoplasm and had a color of light yellow to brown. Magnification, x400. RGS5, regulator of G-protein signaling 5; rh-ES, recombinant human endostatin; NS, normal saline.

performed RT-qPCR on LLC tumors of NS and rh-ES groups. Compared with the NS group, both RGS5 and CA9mRNA levels in the rh-ES group were significantly lower at days 4 and $6(\mathrm{P}<0.05)$, while there was no significant change between two groups at days 2 and 9 (Figs. 6 and 7).

ELISA further confirms the reduction of RGS5 and CA9 protein levels in tumor cells at days 4 and 6 of treatment, but not at days 2 and 9. To further confirm the effect of rh-ES, ELISA was performed on LLC tumors of NS and rh-ES groups. Compared with the NS group, both RGS5 and CA9 protein levels in rh-ES group were significantly lower at days 4 and $6(\mathrm{P}<0.05)$, while there was no significant change between two groups at days 2 and 9 (Figs. 8 and 9 and Tables IV and V).

\section{Discussion}

The use of rh-ES as an antiangiogenic drug in advanced NSCLC has been suggested by the Chinese version of the NCCN guidelines (13). Besides lung cancer (14), studies and clinical trials have demonstrated the anticancer effect of rh-ES in other cancers such as melanoma, nasopharyngeal and esophageal cancer (15-17). These studies have reported that rh-ES can cause transient normalization of tumor vasculature. Consistent with previous studies, using an LLC tumor model, the authors demonstrated that the reduction of microvessel marker CD31 caused by rh-ES occurs between days 4 and 6 following the start of rh-ES treatment, and is reversed at day 9 or even earlier, demonstrating that the vascular normalization of LLC tumors caused by rh-ES is transient and reversible. Based on the 'vascular normalization window' hypothesis proposed by Jain (3), the time window ranging from day 4 to day 6 would be the best scheduling time for a combination therapy using rh-ES and chemo- or radio-therapy.

In the past decade, vast evidence has emerged in support of the 'normalization window' hypothesis for tumor angiogenesis. Molecular mechanisms have been investigated to understand this process. Among these mechanisms, VEGF is demonstrated to be a key regulator for angiogenesis. Although there is no doubt concerning the anti-angiogenic effect of rh-ES, the molecular mechanism for this effect still remains unclear. Different research groups have demonstrated that rh-ES can reduce the expression levels of vascular endothelial growth factor (VEGF), indicating that VEGF may play an important

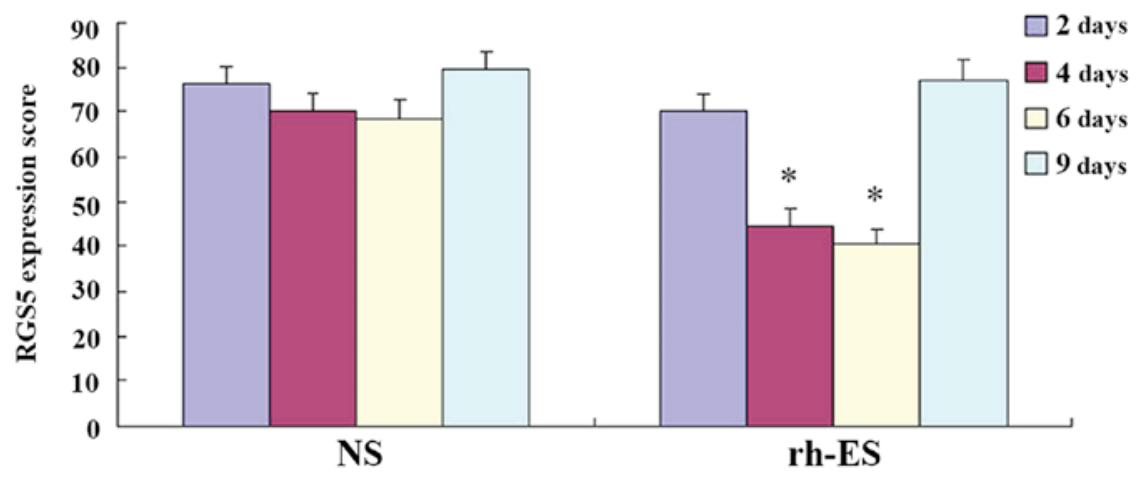

Figure 3. Quantification of RGS5 immunoreactivity. The expression of RGS5 at days 4 and 6of rh-ES-treated Lewis lung cancer tumors demonstrated a significant reduction compared with NS LLC tumors, whilst there was no significant difference at days 2 and 9 between the two groups. ${ }^{*}<0.05$ vs. NS. RGS5, regulator of G-protein signaling 5; rh-ES, recombinant human endostatin; NS, normal saline. 


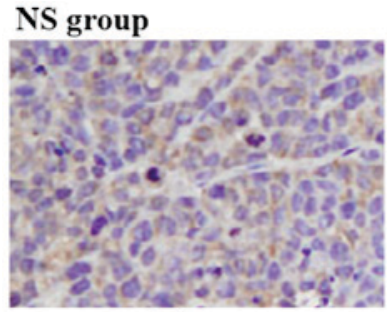

2 days

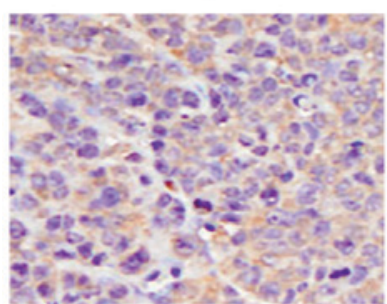

6 days

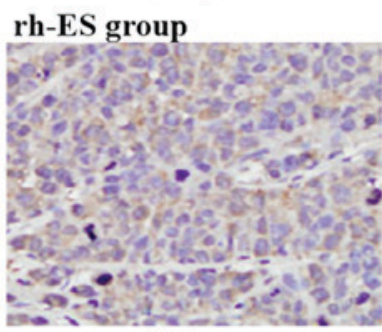

2 days

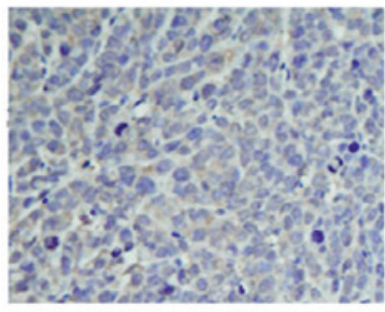

6 days

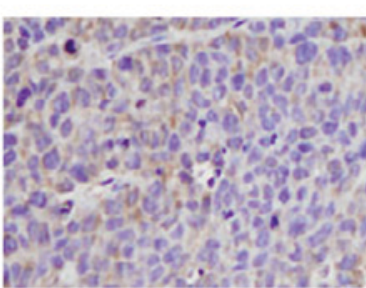

4 days

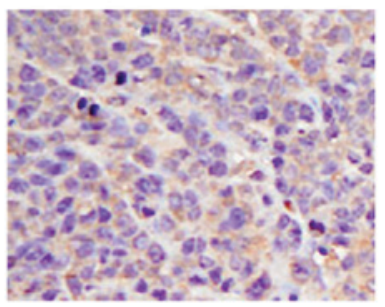

9 days

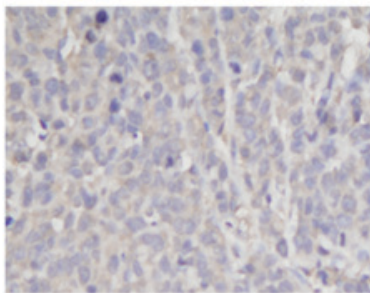

4 days

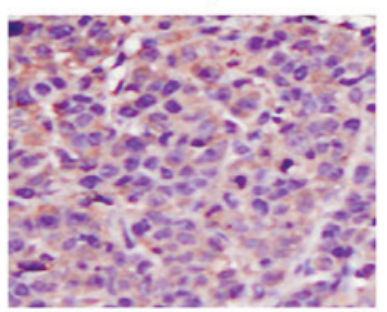

9 days
Figure 4. Representative images showing CA9 (a marker for tumor hypoxia) immunohistochemistry staining in Lewis lung cancer tumors following treatment with rh-ES or NS for days 2, 4, 6 and 9. The positive CA9 signals were located on the cell membrane and had a color of brown. Magnification, x400. CA9, carbonic anhydrase 9; rh-ES, recombinant human endostatin; NS, normal saline.

role in rh-ES-induced vascular normalization $(14,16)$. Aside from this, the general understanding of the effect of rh-ES has been limited. The current findings reported that RGS5 and CA9 were reduced by rh-ES during the "vascular normalization window' are striking. To the best of the authors' knowledge, this is the first direct evidence to present the roles of RGS5 and CA9 in the antiangiogenic effect of rh-ES.

Rgs5 has been identified as a master gene responsible for the abnormal vasculature in mouse tumors. Loss of Rgs 5 can cause vascular normalization and a consequent reduction in tumor hypoxia and vessel leakiness, leading to enhanced influx of immune effector cells into the tumor (18). However, the relationship between RGS5 and VEGF is not completely understood. On one hand, RGS5 can be triggered by tumor vasculature and sustained by VEGF-rich proangiogenic microenvironment (19). One the other hand, RGS5 can antagonize the angiogenic effect of VEGF through the p38 signaling
Table II. Expression of regulator of G-protein signaling 5 in Lewis lunch cancer at different time-points.

\begin{tabular}{lccc}
\hline Group & Time & $\begin{array}{c}\text { Observed } \\
\text { fields }(\mathrm{n})\end{array}$ & $\begin{array}{c}\text { Labeling } \\
\text { index }(\%)\end{array}$ \\
\hline NS & 2 & 5 & $76.13 \pm 4.13$ \\
& 4 & 5 & $70.24 \pm 4.08$ \\
& 6 & 5 & $68.66 \pm 3.76$ \\
rh-ES & 9 & 5 & $79.60 \pm 3.99$ \\
& 2 & 5 & $70.02 \pm 4.03$ \\
& $4^{\mathrm{a}}$ & 5 & $44.77 \pm 3.41$ \\
& $6^{\mathrm{a}}$ & 5 & $40.49 \pm 3.38$ \\
& 9 & 5 & $77.04 \pm 4.26$
\end{tabular}

${ }^{\mathrm{a}} \mathrm{P}<0.05$ (Rh-ES vs. NS). RGS5, regulator of G-protein signaling 5; LLC, Lewis lung cancer; NS, normal saline control group; rh-ES, recombinant human endostatin. Values are presented as mean \pm standard deviation.

Table III. Expression of carbonic anhydrase 9 in Lewis lung cancer at different time points.

\begin{tabular}{lccc}
\hline Group & Time & $\begin{array}{c}\text { Observed } \\
\text { fields (n) }\end{array}$ & $\begin{array}{c}\text { Labeling } \\
\text { index (\%) }\end{array}$ \\
\hline NS & 2 & 5 & $90.10 \pm 3.85$ \\
& 4 & 5 & $78.98 \pm 4.98$ \\
& 6 & 5 & $83.62 \pm 3.68$ \\
rh-ES & 9 & 5 & $80.15 \pm 3.96$ \\
& 2 & 5 & $73.09 \pm 4.19$ \\
& $4^{\mathrm{a}}$ & 5 & $30.68 \pm 3.95$ \\
& $6^{\mathrm{a}}$ & 5 & $35.87 \pm 3.70$ \\
& 9 & 5 & $68.81 \pm 4.92$ \\
\hline
\end{tabular}

${ }^{\mathrm{a}} \mathrm{P}<0.05$ (Rh-ES vs. NS). CA9, carbonic anhydrase 9; LLC, Lewis lung cancer; NS, normal saline; rh-ES, recombinant human endostatin. Values are presented as mean \pm standard deviation.

pathway (20). Whether the reduction of RGS5 by rh-ES is mediated by VEGF or is independent of VEGF needs to be further investigated. The presented tumor model provides the best in vivo system for such a follow-up study to better the understanding of the molecular mechanism involved in the anti-angiogenic effect of rh-ES.

Different to RGS5, the relationship between CA9 and VEGF is clearer. The regulation of CA9 is differential from the regulation of VEGF in hypoxic conditions, which has been demonstrated in bladder cancer (21). By using this system, the authors will be able to examine whether the rh-ES-induced CA9 reduction is independently of VEGF in future studies.

In conclusion, the authors have provided evidence that rh-ES can cause transient and reversible tumor vascular normalization, optimizing the schedule of combination therapy in human tumors. Most importantly, the novel findings that the 


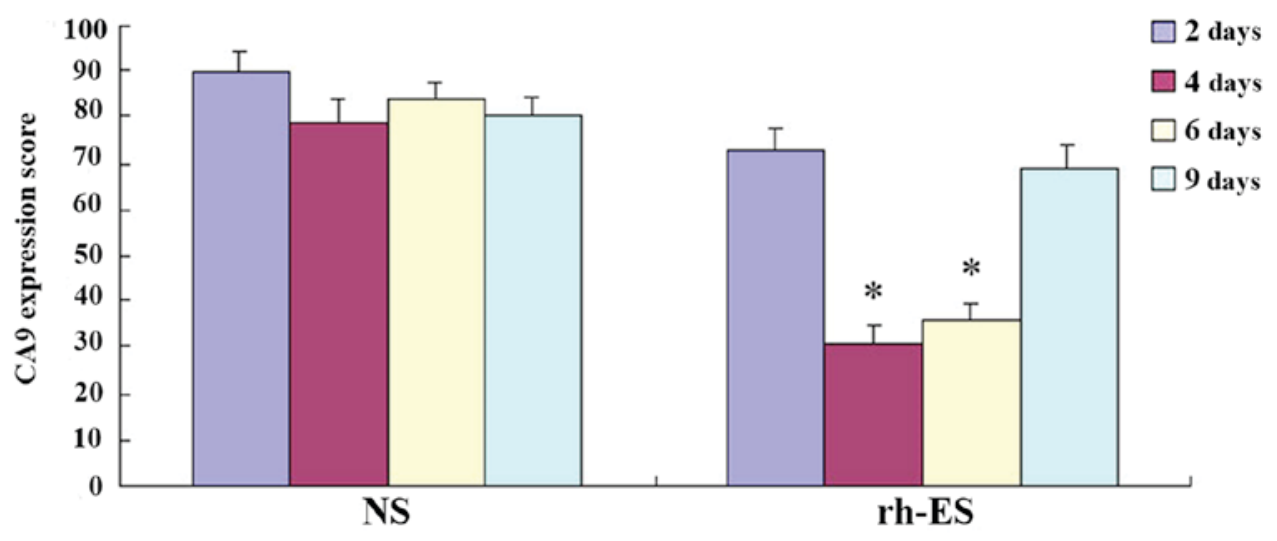

Figure 5. Quantification of CA9 immunoreactivity. The expression of CA9 at days 4 and 6 of rh-ES-treated Lewis lung cancer tumors presented a significant reduction compared with NS LLC tumors, whilst there was no significant difference at days 2 and 9 between the two groups. "P<0.05 vs. NS. CA9, carbonic anhydrase 9; rh-ES, recombinant human endostatin; NS, normal saline.

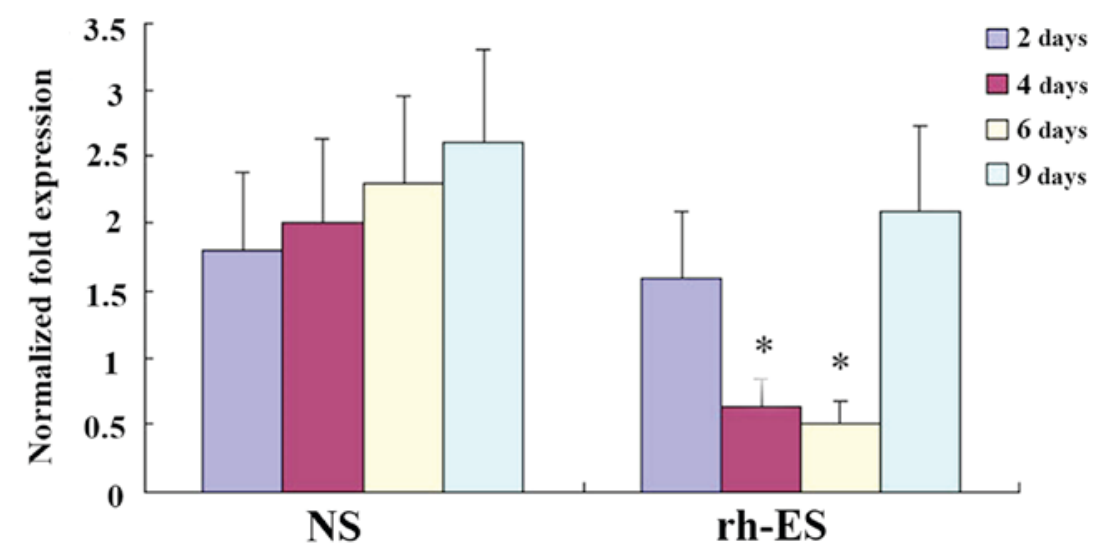

Figure 6. Reverse transcription-quantitative polymerase chain reaction demonstrating RGS5 mRNA levels in rh-ES and NSLLC tumors. RGS5 mRNA levels at days 4 and 6 of rh-ES-treated LLC tumors presented a significant reduction compared with saline-treated LLC tumors while there was no significant difference at days 2 and 9 between the two groups. "P<0.05 vs. NS. RGS5, regulator of G-protein signaling 5; rh-ES, recombinant human endostatin; LLC, Lewis lung cancer; NS, normal saline.

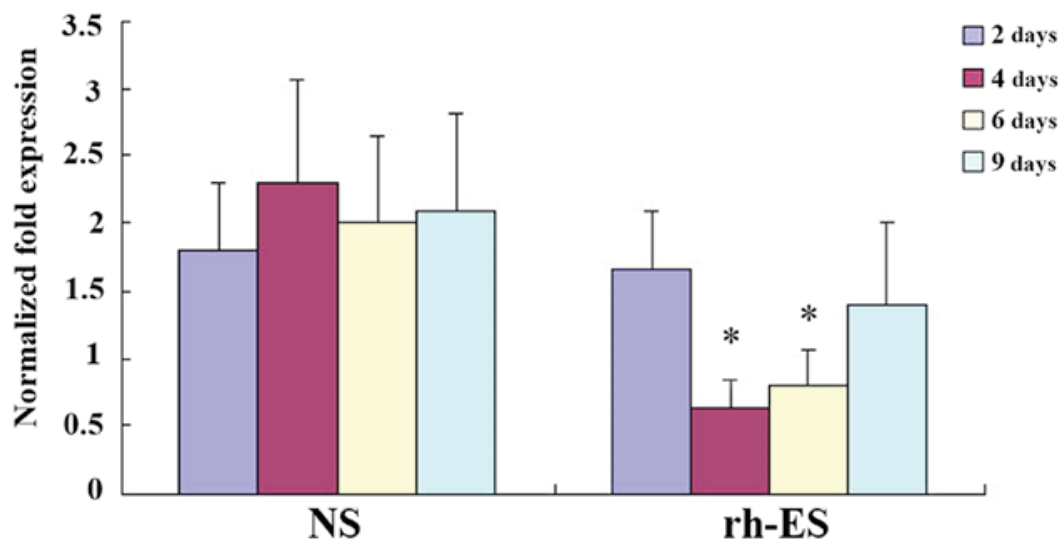

Figure 7. Reverse transcription-quantitative polymerase chain reaction demonstrating CA9 mRNA levels in rh-ES and NS LLC tumors. CA9 mRNA levels at days 4 and 6 of rh-ES-treated LLC tumors presented a significant reduction compared with saline-treated LLC tumors, whilst there was no significant difference at days 2 and 9 between the two groups. "P<0.05 vs. NS. CA9, carbonic anhydrase 9; rh-ES, recombinant human endostatin; LLC, Lewis lung cancer; NS, normal saline.

expression of RGS5 and CA9 is reduced during the 'vascular normalization window' suggested that RGS5 and CA9 may be used as biomarkers for defining the 'vascular normalization window'.

\section{Acknowledgements}

The present study was supported by technology support program of Science and Technology Department of Sichuan 
Table IV. Expression of regulator of G-protein signaling 5 in Lewis lung cancer by ELISA (ng/ml).

\begin{tabular}{lcccc}
\hline Days following injection $(\mathrm{n})$ & 2 & 4 & 6 & 9 \\
\hline NS & $7.88 \pm 0.72$ & $8.16 \pm 0.88$ & $10.51 \pm 1.44$ & $11.08 \pm 1.54$ \\
Rh-ES & $8.45 \pm 0.94$ & $4.02 \pm 0.68^{\mathrm{a}}$ & $2.98 \pm 0.46^{\mathrm{a}}$ & $10.28 \pm 0.96$
\end{tabular}

${ }^{\text {aP }}<0.05$ (Rh-ES vs. NS). RGS5, regulator of G-protein signaling 5; LLC, Lewis lung cancer; NS, normal saline; rh-ES, recombinant human endostatin. Values are presented as mean \pm standard deviation.

Table V. Expression of carbonic anhydrase 9 in Lewis lung cancer by ELISA (ng/ml).

\begin{tabular}{lcrrr}
\hline Days following injection (n) & 2 & 4 & 6 & 9 \\
\hline NS & $148.12 \pm 7.22$ & $166.56 \pm 5.12$ & $180.51 \pm 5.65$ & $189.28 \pm 7.60$ \\
Rh-ES & $146.45 \pm 6.28$ & $88.63 \pm 6.16^{\mathrm{a}}$ & $83.98 \pm 5.76^{\mathrm{a}}$ & $168.20 \pm 8.02$
\end{tabular}

${ }^{\mathrm{a} P}<0.05$ (Rh-ES vs. NS). LLC, Lewis lung cancer; NS, normal saline; rh-ES, recombinant human endostatin; CA9, carbonic anhydrase 9. Values are presented as mean \pm standard deviation.

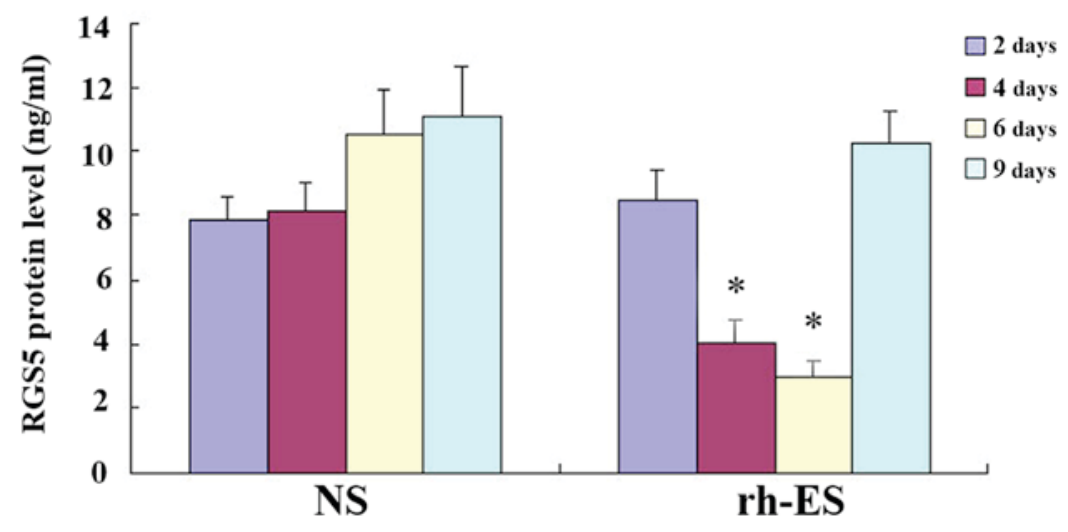

Figure 8. ELISA assay demonstrating RGS5 protein levels in rh-ES and NS LLC tumors. RGS5 protein levels at days 4 and 6 of rh-ES-treated LLC tumors presented a significant reduction compared with saline-treated LLC tumors, whilst there was no significant difference at days 2 and 9 between the two groups. ${ }^{*} \mathrm{P}<0.05$ vs. NS.RGS5, regulator of G-protein signaling 5; rh-ES, recombinant human endostatin; LLC, Lewis lung cancer; NS, normal saline.

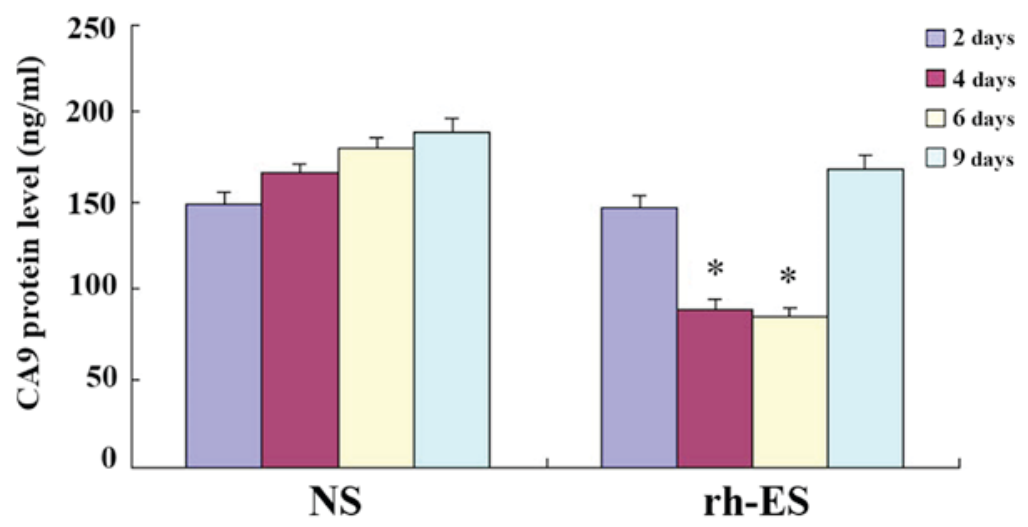

Figure 9. ELISA assay demonstrating CA9 protein levels in rh-ES and NS LLC tumors. CA9 protein levels at days 4 and 6 of rh-ES-treated LLC tumors presented a significant reduction compared with saline-treated LLC tumors, whilst there was no significant difference at days 2 and 9 between the two groups. ${ }^{*} \mathrm{P}<0.05$ vs. NS.CA9, carbonic anhydrase 9; rh-ES, recombinant human endostatin; LLC, Lewis lung cancer; NS, normal saline.

Province (grant no. 2014SZ0020-7), the Department of Science and Technology Innovation Talent Engineering
Projects in Sichuan Province (grant no. 20133007), Sichuan Province Health Department of Scientific Research Projects 
(grant no. 130466), Nanchong Technology Bureau Application Technology and Development of Capital Projects (grant no. 13A0061) and doctoral startup fund of North Sichuan Medical College (grant no. CBY15-QD09).

\section{References}

1. Winkler F, Kozin SV, Tong RT, Chae SS, Booth MF, Garkavtsev I, $\mathrm{Xu}$ L, Hicklin DJ, Fukumura D, di Tomaso E, et al: Kinetics of vascular normalization by VEGFR2 blockade governs brain tumor response to radiation: Role of oxygenation, angiopoietin-1, and matrix metalloproteinases. Cancer Cell 6: 553-563, 2004.

2. Lin MI and Sessa WC: Antiangiogenic therapy: Creating a unique 'window' of opportunity. Cancer Cell 6: 529-531, 2004

3. Jain RK: Normalizing tumor vasculature with anti-angiogenic therapy: A new paradigm for combination therapy. Nat Med 7: 987-989, 2001.

4. Welén K, Jennbacken K, Tesan T and Damber JE: Pericyte coverage decreases invasion of tumour cells into blood vessels in prostate cancer xenografts. Prostate Cancer Prostatic Dis 12: 41-46, 2009.

5. Faraone D, Aguzzi MS, Toietta G, Facchiano AM, Facchiano F, Magenta A, Martelli F, Truffa S, Cesareo E, Ribatti D, et al: Platelet-derived growth factor-receptor alpha strongly inhibits melanoma growth in vitro and in vivo. Neoplasia 11: 732-742, 2009.

6. Manzur M and Ganss R: Regulator of G protein signaling 5: A new player in vascular remodeling. Trends Cardiovasc Med 19: 26-30, 2009.

7. Park SY, Kim YJ, Gao AC, Mohler JL, Onate SA, Hidalgo AA, Ip C, Park EM, Yoon SY and Park YM: Hypoxia increases androgen receptor activity in prostate cancer cells. Cancer Res 66: 5121-5129, 2006.

8. Yasuda H: Solid tumor physiology and hypoxia-induced chemo/radio-resistance: Novel strategy for cancer therapy: Nitric oxide donor as a therapeutic enhancer. Nitric Oxide 19: 205-216, 2008.

9. Bussink J, Kaanders JH and van der Kogel AJ: Tumor hypoxia at the micro-regional level: Clinical relevance and predictive value of exogenous and endogenous hypoxic cell markers. Radiother Oncol 67: 3-15, 2003.

10. O'Reilly MS, Boehm T, Shing Y, Fukai N, Vasios G, Lane WS, Flynn E, Birkhead JR, Olsen BR and Folkman J: Endostatin: An endogenous inhibitor of angiogenesis and tumor growth. Cell 88 : 277-285, 1997.

11. Rahman MA, Dhar DK, Yamaguchi E, Maruyama S, Sato T, Hayashi H, Ono T, Yamanoi A, Kohno H and Nagasue N: Coexpression of inducible nitric oxide synthase and COX-2 in hepatocellular carcinoma and surrounding liver: Possible involvement of COX-2 in the angiogenesis of hepatitis $\mathrm{C}$ virus-positive cases. Clin Cancer Res 7: 1325-1332, 2001.
12. Livak KJ and Schmittgen TD: Analysis of relative gene expression data using real-time quantitative PCR and the 2(-Delta Delta C(T)) method. Methods 25: 402-408, 2001.

13. Shi Y, Sun Y, Yu J, Ding C, Wang Z, Wang C, Wang D, Wang C, Wang Z, Wang M, et al: China experts consensus on the diagnosis and treatment of advanced stage primary lung cancer (2016 version). Asia Pac J Clin Oncol 13: 87-103, 2017.

14. Li N, Zheng D, Wei X, Jin Z, Zhang C and Li K: Effects of recombinant human endostatin and its synergy with cisplatin on circulating endothelial cells and tumor vascular normalization in A549 xenograft murine model. J Cancer Res Clin Oncol 138: 1131-1144, 2012.

15. Cui C, Mao L, Chi Z, Si L, Sheng X, Kong Y, Li S, Lian B, Gu K, Tao M, et al: A phase II, randomized, double-blind, placebo-controlled multicenter trial of Endostar in patients with metastatic melanoma. Mol Ther 21: 1456-1463, 2013.

16. Zhu H, Yang X, Ding Y, Liu J, Lu J, Zhan L, Qin Q, Zhang H, Chen X, Yang Y, et al: Recombinant human endostatin enhances the radioresponse in esophageal squamous cell carcinoma by normalizing tumor vasculature and reducing hypoxia. Sci Rep 5: 14503, 2015.

17. Peng F, Xu Z, Wang J, Chen Y, Li Q, Zuo Y, Chen J, Hu X, Zhou Q, Wang Y, et al: Recombinant human endostatin normalizes tumor vasculature and enhances radiation response in xenografted human nasopharyngeal carcinoma models. PLoS One 7: e34646, 2012.

18. Hamzah J, Jugold M, Kiessling F, Rigby P, Manzur M, Marti HH, Rabie T, Kaden S, Gröne HJ, Hämmerling GJ, et al: Vascular normalization in Rgs5-deficient tumours promotes immune destruction. Nature 453: 410-414, 2008.

19. Silini A, Ghilardi C, Figini S, Sangalli F, Fruscio R, Dahse R, Pedley RB, Giavazzi R and Bani M: Regulator of G-protein signaling 5 (RGS5) protein: A novel marker of cancer vasculature elicited and sustained by the tumor's proangiogenic microenvironment. Cell Mol Life Sci 69: 1167-1178, 2012.

20. Jin Y, An X, Ye Z, Cully B, Wu J and Li J: RGS5, a hypoxia-inducible apoptotic stimulator in endothelial cells. J Biol Chem 284: 23436-23443, 2009

21. Turner KJ, Crew JP, Wykoff CC, Watson PH, Poulsom R, Pastorek J, Ratcliffe PJ, Cranston D and Harris AL: The hypoxia-inducible genes VEGF and CA9 are differentially regulated in superficial vs invasive bladder cancer. Br J Cancer 86: 1276-1282, 2002. 